\title{
Spectral Properties of Rodamine B dissolved in Chloroform
}

\author{
Ali Hadi Al-Hamdani ${ }^{(*)}$, RajaaNader(**), Rafah Abdul Hadi(**) \\ * University of Technology- Laser \&Optoelectronics Engineering Department \\ ** University of Baghdad. College of science for women - physics department
}

\begin{abstract}
The spectral properties (absorption and fluorescence) of Rhodamine B dye have been studied. This type of dye belong to the Xanthenes family and it was dissolved in chloroform with different concentrations $\left(3 \times 10^{-5}, 8 \times 10^{-5}, 8 \times 10^{-4}, 5 \times 10^{-4}, 2 \times 10^{-4}\right.$ and $2 \times 10^{-3}$ mole/liter $)$ at room temperature. The achieved results have been pointed out there are a blue shift in the absorption spectra and red shift in the fluorescence spectra as the concentration increased. The quantum efficiency of the dissolved RB in chloroform were $(53 \%, 58 \%, 67 \%, 76 \%$, $78 \%$ and $89 \%)$ for the above concentrations respectively.It has been noticed that the quantum efficiency decreased as a function of concentration.There is a good consistency of the behavior of the fluorescence and absorption spectra with Beer.
\end{abstract}

Keywords: Fluorescence, Absorbance, Rhodamine B, Quantum efficiency .

\section{Introduction:}

Laser dyes can usually be used for a much wider range of wavelengths. The wide bandwidth makes them particularly suitable for tunable lasers and pulsed lasers. Moreover, the dye can be replaced by another type in order to generate different wavelengths with the same laser, although this usually requires replacing other optical components in the laser as well. Dye lasers were independently discovered by P. P. Sorokin and F. P. Schäfer (and colleagues) in 1966[1,2].In addition to the usual liquid state, dye lasers are also available as solid state dye lasers (SSDL). SSDL use dye-doped organic matrices as gain medium[3-10].

Many studies focued on Xanthenes family which Rodamine B belong to them because of there higher gain $[11,12]$ and their properties differ with many parameters (like,different solvents, different concentrations,...). The chemical structure of RhodamineBisshown in Figure (1)

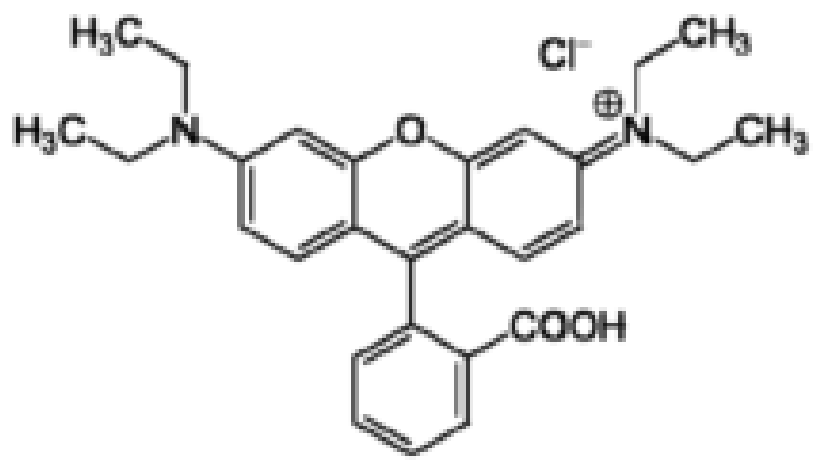

Figure 1: Chemical structure of Rhodamine B [13].

\section{Theoretical Consideration}

The spectrum of the molecular fluorescence $F(v)$ gives the relative fluorescence intensity at wave-number (v́), this is related to the quantum efficiency by the following equation ,[14].

$$
q_{f m}=\int_{0}^{\infty} F\left(v^{\prime}\right) d v^{\prime}
$$

In order to evaluate absolute quantum efficiency, we have to consider both the radiative and non-radiative processes taking place in the medium, therefore

$$
q_{f m}=\frac{K_{f m}}{K_{f m}+\sum K_{d}}=\frac{K_{f m}}{K_{f m}+K_{I C}+K_{I S C}}
$$


Since $\mathrm{K}_{\mathrm{fm}}=1 / \tau \mathrm{fm}$ and $\tau_{\mathrm{f}}=1 / \mathrm{K}_{\mathrm{fm}}+\Sigma \mathrm{K}_{\mathrm{d}}$

Therefore

$$
q_{f m}=\frac{\tau_{f}}{\tau_{f m}}=\int_{0}^{\infty} F\left(v^{\prime}\right) \quad d v^{\prime}
$$

Also

$q_{\mathrm{FM}}=\frac{\text { Number of quanta emitted }}{\text { Number of quanta absorbed }}$

where

$\square_{\mathrm{m}}$ :-The radiation life time can be calculated using relation as follow

$\frac{1}{\tau_{F M}}=2.88 * 10^{-9} n^{2}\left(v^{\prime 2}\right) \int \varepsilon\left({ }^{\prime} v\right) d v^{\prime}$.

Where

n:refractive index of a medium

$\square$ : wave number at the maximum absorption

$\square \square(\square$ ')d ':the area under the absorption spectrum curve as a function of the wavenumber,[15].

Experimental Work:

The materials used in search

The molecular formula of the $\mathrm{RB}$ is $\mathrm{C}_{28} \mathrm{H}_{31} \mathrm{~N}_{2} \mathrm{O}_{3}$ Clandprocessed by the company (HIMEDIA) India .This character belongs to the Xanthenes family and the molecular weight is $479.02 \mathrm{gm} / \mathrm{mol}$.

\section{The solvent :( Chloroform)}

Chloroform is the organic compound with formulaCHCl $\mathrm{CH}_{3}$.

The colorless, sweet-smelling, dense liquid is a trihalomethane, and.The physical properties as showen in the table.

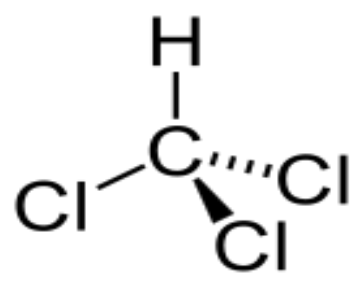

\begin{tabular}{|ll|}
\hline Properties & \\
Molecular formula & $\mathrm{CHCl}_{3}$ \\
Molar mass & $119.38 \mathrm{~g} \mathrm{~mol}^{-1}$ \\
Appearance & Colorless liquid \\
Density & $1.483 \mathrm{~g} / \mathrm{cm}^{3}$ \\
Melting point & $-63.5{ }^{\circ} \mathrm{C}, 210 \mathrm{~K},-82^{\circ} \mathrm{F}$ \\
Boiling point & $61.2{ }^{\circ} \mathrm{C}, 334 \mathrm{~K}, 142{ }^{\circ} \mathrm{F}$ \\
Refractive index & 1.4459 \\
\hline
\end{tabular}

Chloroform Structure, [16].

\section{Instrument used in work:}

-Fluorescence spectrum was measured by a (Spectrofluorometer SL174) by the processor (Elico) -Absorption spectrum was measured by a (SP-8001.UV-visible spectrophotometer) by the processor (Metertechlnc)

The preparation of dye solution withdifferent concentration was taken place by dissolving an appropriate amount of dyepowder in agiven volume of solvent used, according to the relationship:

$$
W=\frac{M_{W} V C}{1000}
$$

Where

$\mathrm{W}$ : Weight of the dissolved dye (gm)

Mw: Molecular weight of the dye

$\mathrm{V}$ : The volume of the solvent $(\mathrm{ml})$

$(\mathrm{gm} / \mathrm{mol})$

$\mathrm{C}$ : The dye concentration $(\mathrm{mol} / \mathrm{l})$ 
The prepared solutions were diluted according to the following equation:-

$\mathrm{C} 1 \mathrm{~V} 1=\mathrm{C} 2 \mathrm{~V} 2$

Where:

$\mathrm{C} 1$ : primary concentration

C2: new concentration

V1: the volume before dilution

V2: the volume after dilution

\section{Results and Discussion}

During the study of the spectral properties of theRhodamine Bdissolved in the chloroformsolvent thefollowing results can be deduced.Figures(2- 7) show the absorption and fluorescence spectra of the solution with differentconcentration $\left(3 \times 10^{-5}, 8 \times 10^{-5}, 8 \times 10^{-4}, 5 \times 10^{-4}, 2 \times 10^{-4}\right.$ and $\left.2 \times 10^{-3} \mathrm{~mol} / \mathrm{liter}\right)$ respectively. The maximum wavelength of the fluorescence and absorption spectra was shown to be depend on the concentration of the dye solution, as shown the figures.Table (1) shows the absorption, fluorescence peaks, and the quantum efficiency of the dye and StokeShift.At dilute of dye, $3 \times 10^{-5} \mathrm{~mol} / \mathrm{litre}$, the quantum efficiency appears high while the maximum wavelength shift (Stoke Shift) is $132 \mathrm{~nm}$, the quantum yield of the dye solution decrease as the dye concentration increase, show theFigure (8) .Increasing the concentration of the dye solution resulted in red shifting of the fluorescence wavelength to higher wavelengths.

\section{Conclusion}

From the observed results of studying the fluorescence and absorption spectra of Rhodamine B dye dissolved in chloroform one can conclude the following:-

1. The increase in dye concentration showed an increase in relative intensity for both spectra, which is in agreement with Beer - Lambrt law.

2. The Stock's shift for fluorescence spectrum of the RB dye dissolved in chloroform is toward the longer wavelength (low energy) as the concentration increased.

3. It must be noted that the quantum yield of the dye solution decrease as the dye concentration increase.

4. As the dye concentration increase the interference region between the fluorescence and absorption spectra have been increased which produced a decrease in Stock's shift.

Table(1): Absorption and Fluorescence peaks and the Quantum efficiency of RB

\begin{tabular}{|r|r|r|r|r|}
\hline $\begin{array}{r}\text { Concentration } \\
\text { mole/Liter }\end{array}$ & $\begin{array}{r}\square_{\mathbf{a} b .} \mathbf{m a x} \\
\mathbf{n m}\end{array}$ & $\begin{array}{l}\square_{\text {flum }} \mathbf{m a x} \\
\mathbf{n m}\end{array}$ & $\begin{array}{r}\text { Stoke } \\
\text { Shift } \\
\mathbf{N m}\end{array}$ & $\begin{array}{r}\text { Quantum Efficiency } \\
\mathbf{q}_{\mathbf{f m}}\end{array}$ \\
\hline $8 \times 10^{-5}$ & 553.82 & 597 & 44.82 & $89 \%$ \\
\hline $3 \times 10^{-5}$ & 552.48 & 608 & 56.48 & $78 \%$ \\
\hline $8 \times 10^{-4}$ & 541.84 & 640 & 98.16 & $76 \%$ \\
\hline $5 \times 10^{-4}$ & 548.56 & 645 & 93.5 & $67 \%$ \\
\hline $2 \times 10^{-4}$ & 551.44 & 647 & 132 & $58 \%$ \\
\hline $2 \times 10^{-3}$ & 515.69 & & & $53 \%$ \\
\hline
\end{tabular}

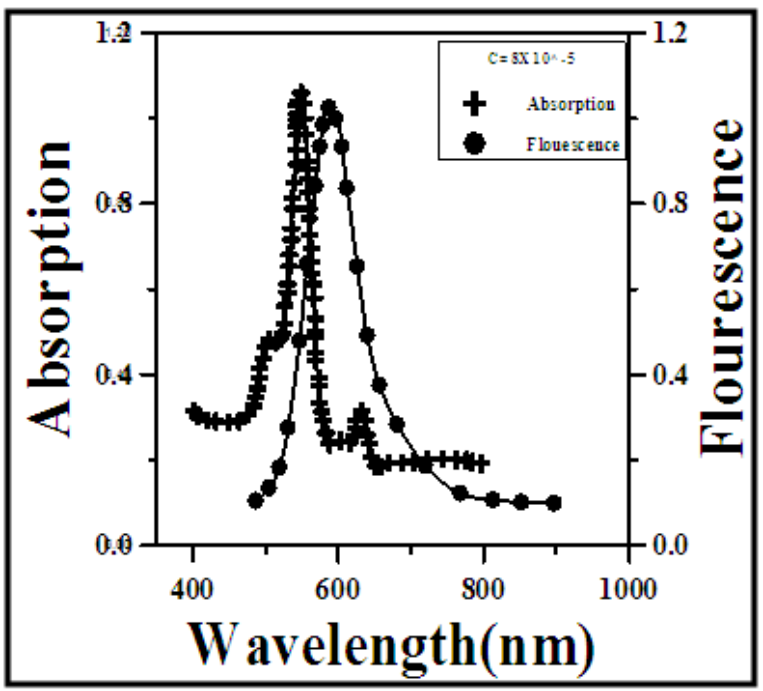

Figure (2) The absorption and Fluorescence spectrum of RB dye concentration $8 \times 10-5 \mathrm{~mol} / \mathrm{L}$. 


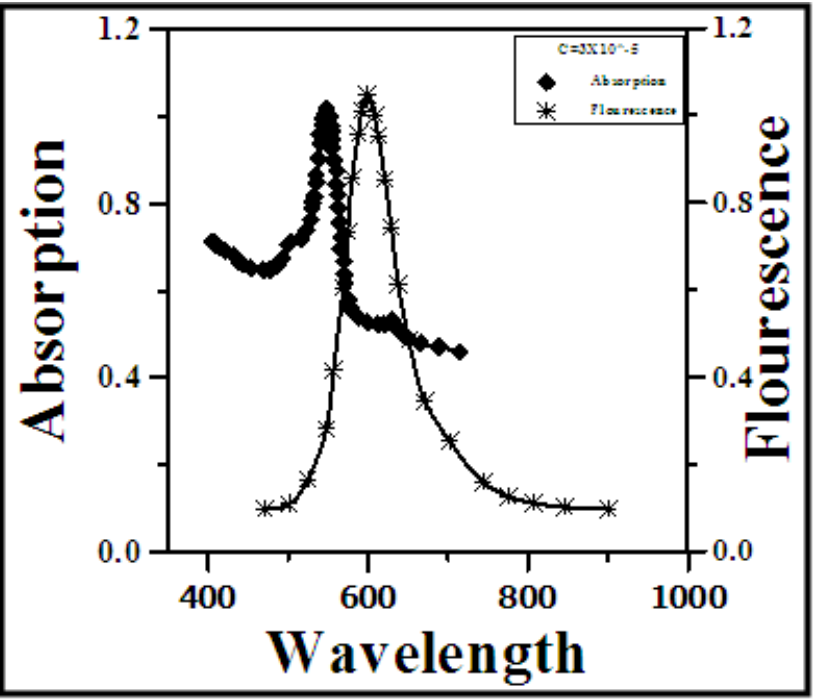

Figure (3) The absorption and Fluorescence spectrum of RBdye for concentration 3×10-5mol/L

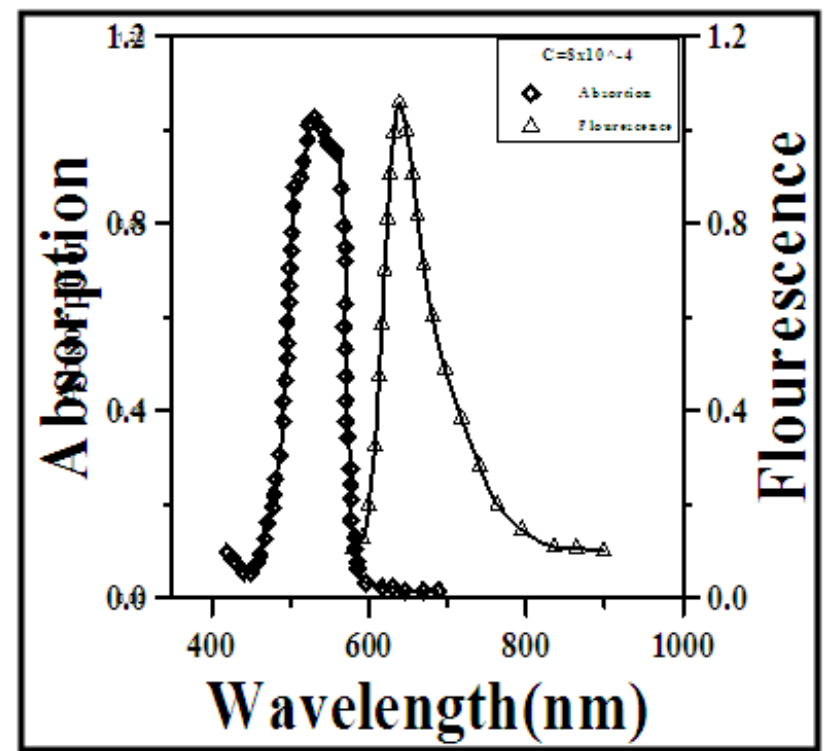

Figure (4) The absorption and Fluorescence spectrum of RB dye for concentration $8 \times 10-4 \mathrm{~mol} / \mathrm{L}$

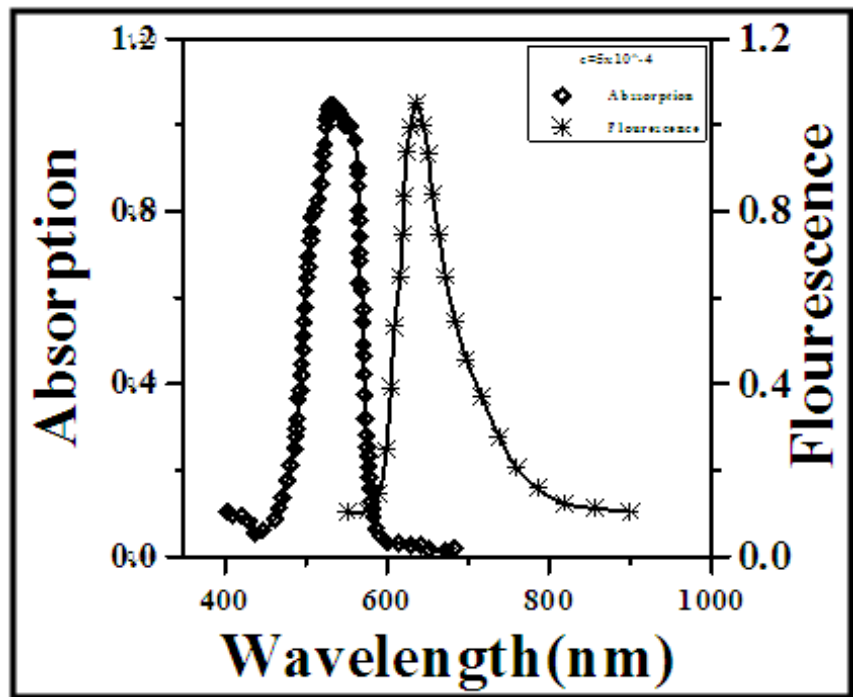

Figure (5) The absorption and Fluorescence spectrum of RB dye for concentration $5 \times 10-4 \mathrm{~mol} / \mathrm{L}$ 


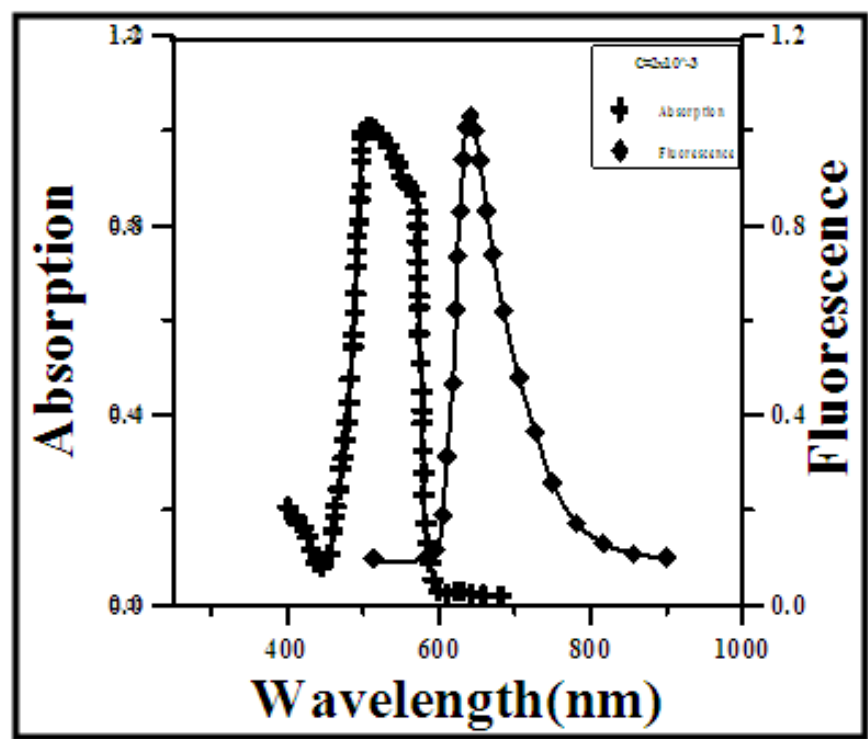

Figure (6) The absorption and Fluorescence spectrum of RBdye for concentration $2 \times 10-4 \mathrm{~mol} / \mathrm{L}$

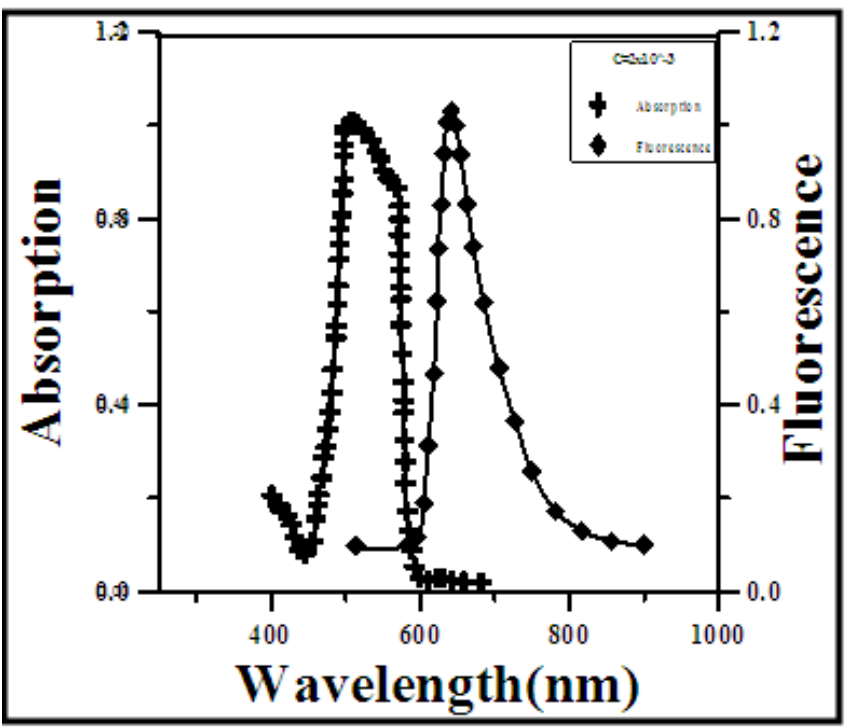

Figure (7) The absorption and Fluorescence spectrum of RBdye for concentration $2 \times 10-3 \mathrm{~mol} / \mathrm{L}$

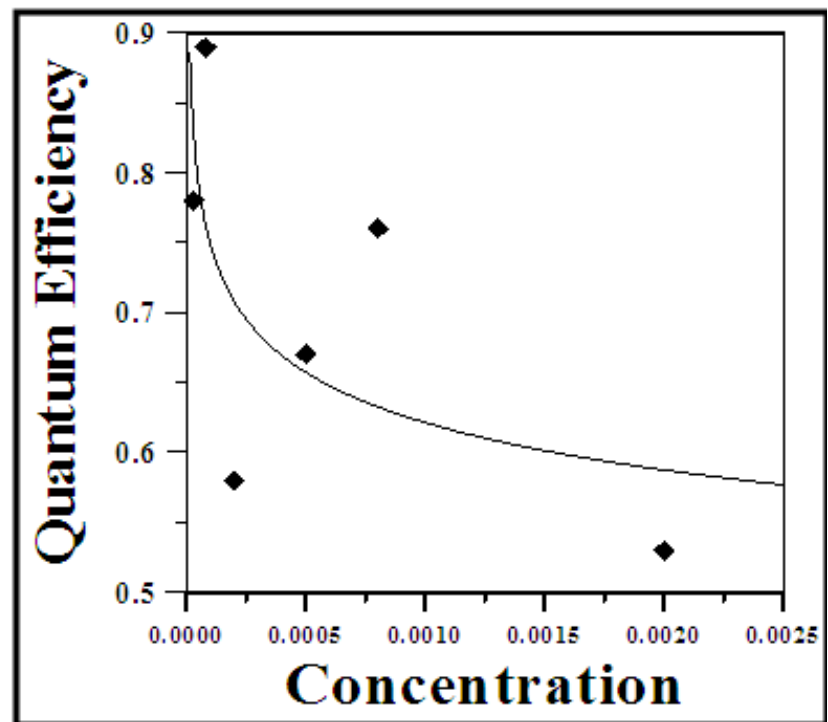

Figure (8)The relation between Quantum Efficiency and the Concentration of RhB dye 


\section{References:}

[1]. Schäfer,F. P., (Ed.), (1990). Dye Lasers (Springer-Verlag, Berlin).

[2]. Duarte, F. J., and Hillman, L. W., (Eds.),(1990).Dye Laser Principles (Academic, New York ).

[3]. Alekseev,N. N., GorelenKo, A.Y.,Kalasho, I. I., andSeroua, V. H.,(1988). J. Appl. Spectry, Vol.49,pp.1232.

[4]. Costela,A.,MerenoG., and I et al., Journal of applid physics, Vol.83, 1998.

[5]. Ali H. Al-Hamdani, Adnan Saleh, (2010), "Fluorescence Efficiency of Rhodamine 6G Doped PMMA “, Journal of materials sciences and engineering (2010) Vol.4 N0. 4

[6]. Ali Hadi Al-Hamdani (*), ShaimaKhyioon ,Rafa,abdulHadi, Calculation the Quantum Efficiency of Mixture of Rhodamine(C and 6G) dyes dissolved in Chloroforme مؤذ مر وق ائ

[7]. Ali H. Al_Hamdani, saad Al-Anbaki, Hussein Ali Hadi, " Experimental study of the performance of fluorescent concentrator "Engineering \& Technology, Journal Vol. ؟ ,No. $؟(2013)$.

[8]. Ali H. Al_Hamdani."Z-Scan measurements of optical nonlinearities for ( $3 \mathrm{GO}$ ) dye". International journal of nanoelectronics and materials science,6(2013)139-

[9]. Ali H. Al_Hamdani, SPECTRAL PROPERTIES OF LIQUID SAMPLES OF HODAMINE (3GO) DYE DISOLVED IN CHLOROFORM". IRE.CH.E. VOL.5 NO.5 2013

[10]. Ali H. Al-Hamdani, Rafa, H. Raja Nader." Study the spectral properties of rodamin 6G dyes doped polymer (PMMA) dissolved in

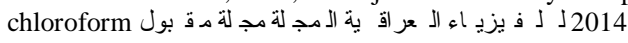

[11]. IsraHadi,(2009). "Study the effect of the solvent and concentration on the absorption and the fluorescence spectume of coumarine-2 dye laser' 'Thesis University Of Baghdad.

[12]. Bindhu, C. V. and Harilal, S. S., (2001). Effect of excitation source on the quantum yield measurements of RhodamineB using thermal lens technique.Anal. Sci., 17, 141-144.

[13]. Fikry, M., Omar, M. M., and Lotfi, Z. I., (2009).Effect of host medium on the fluorescence emission intensity of Rhodamine B in liquid and solid phase. J. Fluores., 19, 741 -746.

[14]. Yarive A., 1975,"Quantum Electronics",John Wiley and Sonss INC.,24.

[15]. ShaimaKhyioon(2012)"Spectroscopic Study of Polymer (PMMA)Dopped with bothRhodamin Dyes RC, RB and their mixture" Thesis University Of Baghdad.

[16]. M. Rossberget al. "ChlorinatedHydrocarbons" in Ullmann's Encyclopedia of Industrial Chemistry, 2006. 